\title{
Absence of a Crystal Direction Regime in which Sputtering Corresponds to Amorphous Material
}

\author{
K. Schlueter@ \\ Max-Planck-Institut für Plasmaphysik, Boltzmannstrasse 2, D-85748 Garching, Germany and \\ Fakultät für Maschinenwesen, Technische Universität München, D-85748 Garching, Germany \\ K. Nordlund $\odot$ \\ Department of Physics, University of Helsinki, P.O. Box 43, FIN-00014 Helsinki, Finland \\ G. Hobler® \\ Institute of Solid-State Electronics, TU Wien, Gußhausstraße 25-25a, A-1040 Wien, Austria \\ M. Balden (1) \\ Max-Planck-Institut für Plasmaphysik, Boltzmannstrasse 2, D-85748 Garching, Germany \\ F. Granberg $\odot$ and O. Flinck \\ Department of Physics, University of Helsinki, P.O. Box 43, FIN-00014 Helsinki, Finland \\ T. F. da Silva@ \\ Physics Institute of University of São Paulo, Rua do Matão 1371, 05508-090 São Paulo, Brazil \\ R. Neu(1) \\ Max-Planck-Institut für Plasmaphysik, Boltzmannstrasse 2, D-85748 Garching, Germany and \\ Fakultät für Maschinenwesen, Technische Universität München, D-85748 Garching, Germany
}

(Received 24 July 2020; accepted 7 October 2020; published 23 November 2020)

\begin{abstract}
Erosion of material by energetic ions, i.e., sputtering, is widely used in industry and research. Using experiments and simulations that, independently of each other, obtain the sputter yield of thousands of individual grains, we demonstrate here that the sputter yield for heavy $\mathrm{keV}$ ions on metals changes as a continuous function of the crystal direction. Moreover, we show that polycrystalline metals with randomly oriented grains do not sputter with the same yield as the amorphous material. The key reason for this is attributed to linear collision sequences rather than channeling.
\end{abstract}

DOI: 10.1103/PhysRevLett.125.225502

The effect of the crystal lattice on material properties is a multifarious topic [1-12]. In applications, it may be employed to advantage, or, at least, it must be controlled. In general, the crystal lattice has an impact on many material properties; however, often only the low-index surfaces are investigated [13-18]. Therefore, a systematic study is highly desirable for characterizing properties such as sputtering $[19,20]$ over many different crystal orientations.

Widely used sputter simulation programs such as TRIM [21] or SDTrimsP [22] assume an amorphous target; i.e., the crystal lattice structure is completely ignored [23,24].

Published by the American Physical Society under the terms of the Creative Commons Attribution 4.0 International license. Further distribution of this work must maintain attribution to the author(s) and the published article's title, journal citation, and DOI. Open access publication funded by the Max Planck Society.
Often, the sputter yield of a polycrystalline sample is compared with simulations [25], and the texture of the sample is not taken into account, even though it is well established that polycrystalline samples often have a preferred crystal orientation at the surface [26,27] and the sputter yield is reduced for low-index surface orientations [14-17]. The underlying assumption is that either the average over all directions corresponds to amorphous material [24,28] or there is a wide range of "random" crystal directions for which the yield is the same as in amorphous material.

This assumption is likely due to an assumed analogy to ion implantation. There, the projected ion range usually varies very little away from the major channeling directions. This is illustrated in Fig. 1(a), which shows an inverse pole figure (IPF) of the projected range of $30 \mathrm{keV} \mathrm{Ga}$ in single-crystalline W. An IPF is a stereographic projection of crystal directions. In cubic systems, due to symmetry the 

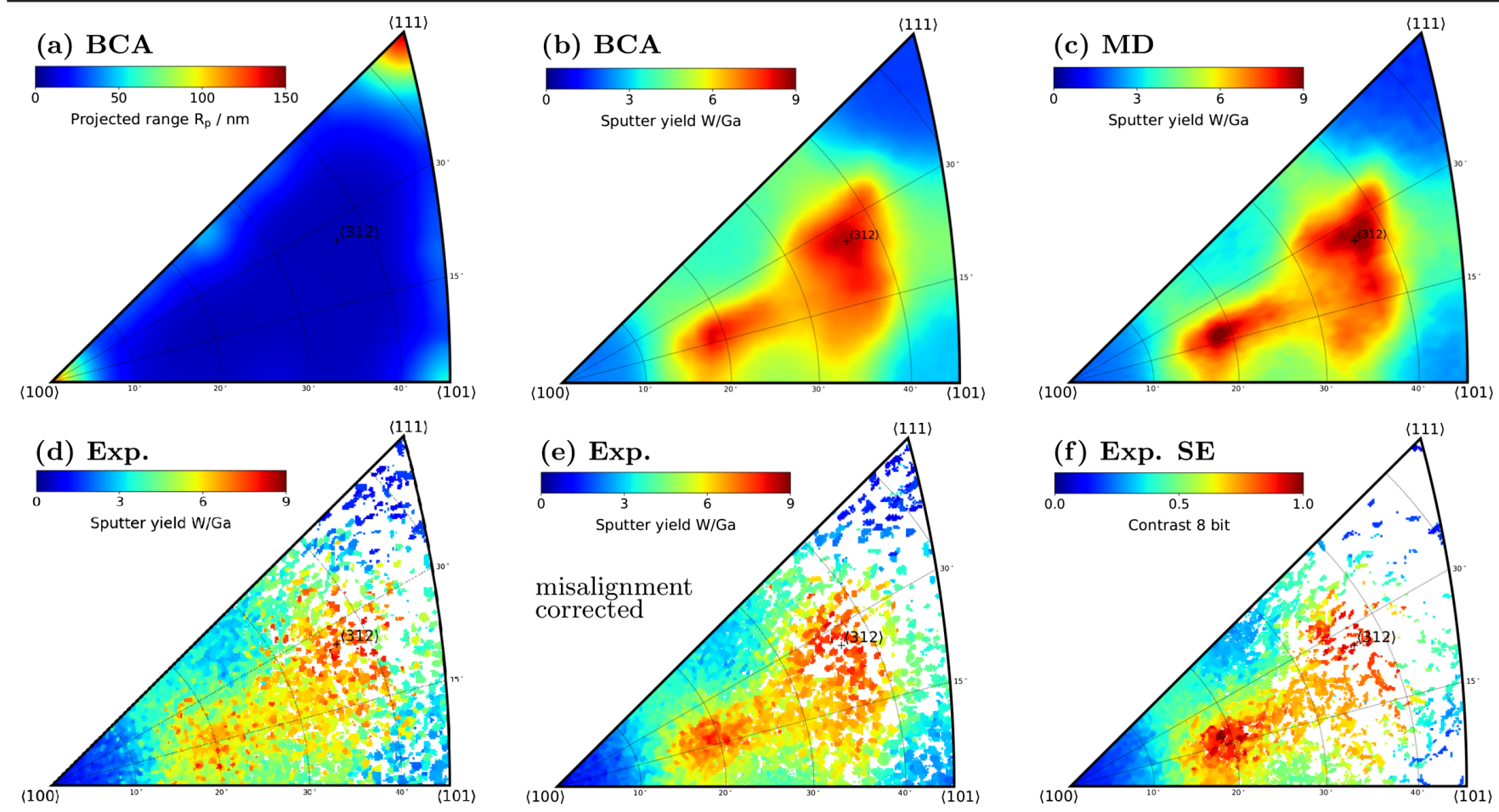

FIG. 1. IPFs for $30 \mathrm{keV} \mathrm{Ga}$ bombardment of W: (a) BCA simulations of the projected range of Ga; (b) BCA simulations of the sputter yield; (c) MD simulations of the sputter yield; (d) experimental sputter yield from four individual measurements; (e) experimental data of (d) corrected by rotation matrix multiplication according to PCA; (f) secondary electron emission. The white areas in the triangles in (d)-(f) represent crystal orientations for which no grains exist in the measured area.

triangle between $\langle 001\rangle,\langle 101\rangle$, and $\langle 111\rangle$ represents the whole angular space. Figure 1(a) shows simulation results obtained with the binary collision approximation (BCA) code IMSIL [29]. The projected ranges are color coded based on an array of $46 \times 46$ implantation simulations for surface orientations covering the orientation triangle. For further details of the simulations, see Supplemental Material [30].

BCA simulations of single-crystal sputtering have been performed in the past [47-49] but only for selected surface orientations. In Fig. 1(b), we show an IPF for the sputter yield obtained by the same IMSIL simulations as used for Fig. 1(a). The $\langle 100\rangle$ and $\langle 111\rangle$ directions have the lowest sputter yield, while they have the largest projected range. However, in contrast to the projected range, the sputter yield changes continuously with crystal orientation, and there is no extended region where it is approximately constant.

In the following, we corroborate this result by molecular dynamics (MD) simulations and experiments using electron backscatter diffraction (EBSD) and scanning electron microscopy (SEM).

For the MD simulations, we developed a new approach to obtain sputter yields for arbitrary crystal orientations with the program MDRANGE, which is based on the recoil interaction approximation (RIA) [50,51]. In principle, MD simulations of the full development of collision cascades (which automatically include heat spike effects [52-56]) could provide the crystal direction dependence of sputtering. However, each full MD simulation is time consuming, and, to get a statistically reliable value for the sputter yield in a single direction, thousands of impacts are needed. Moreover, for obtaining IPFs, easily over thousands of directions are needed. These calculations are computationally prohibitive for full MD simulations. Therefore, MD simulations were performed on two levels. First, the crystal-direction dependence of the energy transfer to recoils in the top $2 \mathrm{~nm}$ of the surface of tungsten was simulated for all crystal directions using MDRANGE. Second, full MD cascade simulations using the PARCAS code [57-59] were performed of eight crystal directions to correlate energy to recoils with the sputter yield [30]. The result illustrates a linear dependence of the sputter yield on the energy to recoils within the statistical uncertainty (see Fig. S3 [30]). The slope of the linear regression obtained from the PARCAS simulations is used to translate the energy to recoils determined by MDRANGE to sputter yield in an IPF, which is shown in Fig. 1(c). Note the almost perfect agreement between $\mathrm{MD}$ and BCA results [Figs. 1(c) and $1(b)$, respectively].

Most previous experimental studies have investigated the sputter yield for some specific low-index crystal orientations only, and some others have measured sputter yield for a few additional orientations [14-17]. To go beyond this 
standard approach, in this work we utilize recent developments in the EBSD technique that allow measuring and generating orientation maps of a polycrystalline sample in a reasonable time [60]. EBSD has been used to study dislocations [61], deformation structures within grains [62], or the crystal-dependent-oxidation behavior [63,64].

EBSD has previously been applied to examine sputter yields for $25 \mathrm{keV} \mathrm{Ga}$ bombardment of Mo [65] yet only for a few surface orientations. More recently, Nagasaki et al. have studied $4 \mathrm{keV}$ Ar bombardment of polycrystalline $\mathrm{Cu}$ and $\mathrm{Ni}$ [66]. Although not discussed, the IPFs shown in that paper indicate a smooth variation of the sputter yield with surface orientation. However, their results have been smoothed due to the relative scarcity of the data, while our raw data have been sharpened by rotation matrix multiplication as described later.

For obtaining the experimental data, hot-rolled polycrystalline tungsten samples were recrystallized to achieve a grain size of around $10 \mu \mathrm{m}$. The samples were polished to achieve flat surfaces with height differences between the grains of less than $50 \mathrm{~nm}$. The tungsten samples were sputtered by focused Ga ion beam (FIB) at $30 \mathrm{keV}$. The grains with the lowest sputter yield have an erosion of around $150 \mathrm{~nm}$, and grains with the highest sputter yield have an 8 times higher erosion, which is measured with a confocal laser scanning microscope (CLSM). Between the sputter experiment and the height measurement, EBSD measurements were performed for determining the crystal orientation. The data of the orientation map and the height data were merged with a PYTHON tool, which was introduced and applied to grain-orientation-dependent oxidation in Ref. [64]. The height data were transformed to sputter yields by using the fluence and the atomic density of the target. Because of the well-known rectangular area irradiated by the scanning FIB, there is a well-defined reference level outside of the sputtered area, and the sputtered depth of each grain is accurately determined. At the end, the sputter yields of ten thousand single-crystal grains from four individual experiments were evaluated for the presented data. These results are visualized in the IPF shown in Fig. 1(d). Note that several grains correspond to each pixel, which represents one crystal direction. Therefore, the median of the sputter yields is reported for each pixel.

The huge data amount opens up the possibility for detailed analysis. A principal component analysis (PCA) is applied on the experimental data [67], to correct a possible misalignment error by the sample mounting or ion beam. A PCA captures the highest variation of the data in a lower dimension, here the IPF. An IPF includes all lattice symmetries and reduces the number of dimensions from three Euler angles to two independent variables characterizing one direction. When the direction is aligned with the ion beam, the highest variation of the data is kept in a lower dimension, because a rotation of the crystal lattice around the ion beam axis does not change the sputter yield; respectively, the ion beam faces the same crystal lattice in every rotation angle. On the EBSD data, rotation matrix multiplications result in a new arrangement of sputter yields in the IPF and the arrangement with the highest variation is desired [Fig. 1(e)].

The PCA was performed on each sputter experiment separately to compensate for the misalignment due to sample mounting or the positioning of the scan area of the FIB beam. The error of around $2^{\circ}$ was corrected by rotation matrix multiplication. The resulting IPF shown in Fig. 1(e) is much clearer than that of the raw data [Fig. 1(d)]. The difference shows that the method is very sensitive to the misalignment error, and, in contrast to common assumptions, errors of the order of $1^{\circ}$ have a significant impact on the data evaluation. Vice versa, the accuracy of the impact angle detection of the ions is better than $1^{\circ}$ (Figs. S11 and S12 [30]).

Comparison of the experimental and simulated data [Figs. 1(b), 1(c), and 1(e)] shows excellent agreement of the dependence of the sputter yield on the crystal direction. It is emphasized that the simulations did not employ any fitting of parameters. The frequency distribution analysis of sputter yields shown in Fig. 2 confirms that the good agreement is not only visual. It also illustrates that the yields vary continuously and there is no single sputter yield value that would be much more common than the other, in contrast to the projected range. Moreover, the fact that the BCA and MD maximum sputter yields are similar indicates that heat spikes do not have a major contribution to sputtering under these irradiation conditions due to the very high melting point of $\mathrm{W}$ [54]. In addition, Fig. 2 shows the values of the sputter yield of amorphous and polycrystalline tungsten, 2.29 and $4.71 \mathrm{~W} / \mathrm{Ga}$, respectively, as

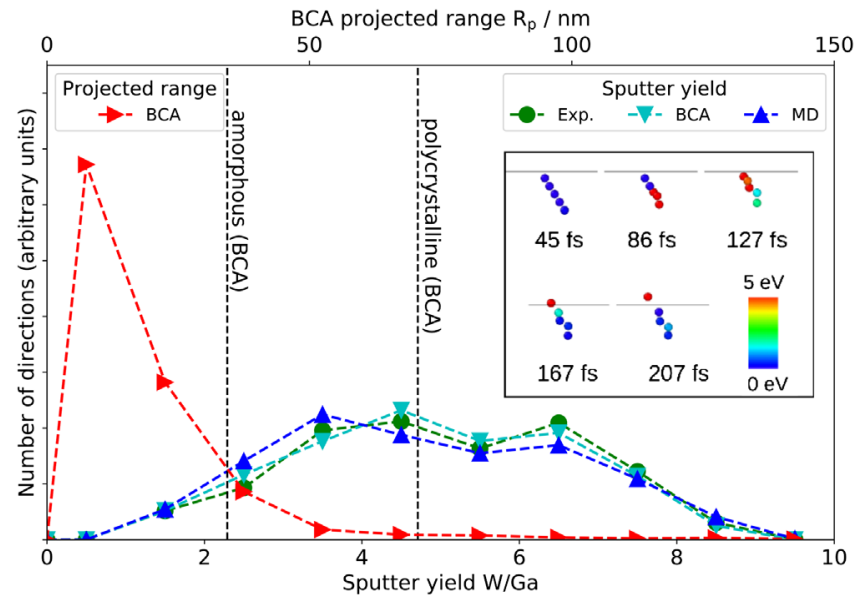

FIG. 2. Number of crystal directions versus sputter yield (green, cyan, and blue lines) and projected range (red line), assuming an isotropic distribution of surface orientations. The average sputter yield of polycrystalline and amorphous $\mathrm{W}$ as obtained by the BCA simulations is indicated by the vertical dashed lines. The inset shows an example of a linear collision sequence, which exists only in crystalline targets, observed in the MD simulations. 
calculated with IMSIL. The discrepancy between the two will be discussed toward the end of the paper.

It is known that the sputter yield correlates with the secondary electron emission $[68,69]$. Therefore, secondary electron emission, caused by the $\mathrm{Ga}^{+}$FIB, was measured with the secondary electron detector of the microscope. The resulting grayscale image was merged with the crystal orientation image to visualize in an IPF, which is shown in Fig. 1(f). The distribution of the secondary electron yield versus the crystal orientation matches the experimental, MD, and BCA sputter yields very well. This demonstrates that secondary electron emission has a linear dependence on the sputter yield over thousands of data points (see Fig. S8 [30]).

In addition, the $\mathrm{Ga}$ implantation due to the crystal orientation was investigated. For that purpose, energy dispersive x-ray spectroscopy (EDX) measurements of the sputtered surface were performed, and the data were merged with the orientation data with the same PYTHON tool as used for the CLSM data [64]. The resulting IPF (Fig. S9 [30]) shows high Ga contents around low-index crystallographic directions where the projected range is large and the sputter yield small. This is to be expected, since both features make it more difficult to remove the implanted ions from the target.

For a detailed investigation of the depth distribution of the channeled Ga ions, FIB-prepared cross sections were made on low- and high-index surfaces for comparing them and verifying the Ga content with EDX. At the surface, the Ga content was around 20 at\% in the $\langle 100\rangle$ and $\langle 111\rangle$ surface orientation. In contrast, at the high-index surfaces, the Ga content was below the detection limit of the EDX. Also, Ga was found down to about $350 \mathrm{~nm}$ below the surface in the $\langle 100\rangle$ and $\langle 111\rangle$ surface orientation. This result is in excellent agreement with MDRANGE and IMSIL simulations, whose electronic stopping power models have been calibrated with independent experimental [70] and theoretical data [71]. They show a maximum range of about $370 \mathrm{~nm}$ for these orientations.

These consistent and accurate experimental results, together with the BCA and MD simulations, convincingly show that there are no extended angular regions with almost constant sputter yield. To support the generality of this conclusion, we have in addition performed BCA simulations for 3 and $300 \mathrm{keV}$ Ga bombardment with qualitatively similar results (see Fig. S1 [30]). In addition, measurements of the dependence of the sputter yield of $\mathrm{Cu}$ on the ion incidence angle for various ion beam conditions $[14,15,28,72]$ may be interpreted as the superposition of a crystal orientation effect similar to that found in the present work and the incidence angle dependence of the sputter yield that is also present for amorphous targets. Thus, our result does not seem to be restricted to heavy ions and the bcc crystal lattice.

We briefly comment on the reasons why there is a continuous variation of the sputter yield with crystal orientation, while there are extended angular regions with almost constant projected ion ranges. Channeling, responsible for the increased projected range along low-index crystallographic directions, is the stable oscillation of trajectories in the open space between atomic rows or planes $[73,74]$. Thus, it takes full effect over an extended depth interval. In contrast, sputtering is a pronounced surface effect, which depends on various processes taking place in the near-surface region $[72,75]$.

Finally, we comment on the difference between the average sputter yields of amorphous and polycrystalline tungsten as indicated in Fig. 2. It has long been known that so-called linear collision sequences $[75,76]$ contribute to sputtering and cause spots in the angular distributions of the sputtered atoms. Since linear collision sequences can only occur in a crystalline material, the large difference in the sputter yields obtained by the BCA simulations using exactly the same physical models with only different target structures provides strong evidence that linear collision sequences contribute significantly to the sputter yield. Also, MD simulations comparing sputtering from amorphous and crystalline tungsten showed a similar difference and exhibited linear collision sequences; see the inset in Fig. 2. Details on the comparison between amorphous and crystalline material yields of the BCA and MD simulations are given in Supplemental Material [30].

In summary, new experimental and simulation approaches are developed for determining the crystal grain-orientation dependence of sputtering. Because of automated data collection processes, a huge amount of experimental data on sputtering can be collected, which, in turn, allows using filter techniques and new analysis methods to be applied on the dataset. This includes the optimization of the data due to the impact angle of the ions. In addition, this technique can be used to detect the impact angle of the ions to the sample surface with an accuracy better than $1^{\circ}$.

The newly developed approaches consistently show that for the system studied the low-index surfaces have more than a factor of 8 lower sputter yield than the high-index surfaces and that there is no continuous wide range of crystal directions that would correspond to the random material assumed in common Monte Carlo BCA codes. Moreover, detailed analysis of the atom collision sequences in the simulations showed that the reason for the large effects is that the physics of sputtering in polycrystalline nonamorphizing materials - such as common bulk elemental metals with high symmetric structure-is fundamentally different from that in amorphous materials.

This work has been carried out within the framework of the EUROfusion Consortium and has received funding from the Euratom research and training programme 20142018 and 2019-2020 under Grant Agreement No. 633053. The views and opinions expressed herein do not necessarily reflect those of the European Commission. 
[1] W. Zhang and K. L. Turner, Sens. Actuators A 122, 23 (2005).

[2] W. Utsumi, H. Saitoh, H. Kaneko, T. Watanuki, K. Aoki, and O. Shimomura, Nat. Mater. 2, 735 (2003).

[3] L. Thomas, F. Lionti, R. Ballou, D. Gatteschi, R. Sessoli, and B. Barbara, Nature (London) 383, 145 (1996).

[4] J. Goldberger, R. He, Y. Zhang, S. Lee, H. Yan, H.-J. Choi, and P. Yang, Nature (London) 422, 599 (2003).

[5] T. Suwada, M. Satoh, K. Furukawa, T. Kamitani, T. Sugimura, K. Umemori, H. Okuno, Y. Endou, T. Haruna, R. Hamatsu et al., Phys. Rev. Spec. Topics-Accelerators Beams 10, 073501 (2007).

[6] A. Stognij, N. Novitskii, and O. Stukalov, Tech. Phys. Lett. 28, 17 (2002).

[7] C. R. Giuliano, Appl. Phys. Lett. 21, 39 (1972).

[8] W. Petasch, B. Kegel, H. Schmid, K. Lendenmann, and H. Keller, Surf. Coat. Technol. 97, 176 (1997).

[9] D. O'kane and K. Mittal, J. Vac. Sci. Technol. 11, 567 (1974).

[10] M. L. Swanson, J. Tesmer, and M. Nastasi, Channeling, in Handbook of Modern Ion Beam Analysis, edited by J. Tesmer, and M. Nastasi (Materials Research Society, Pittsburgh, PA, 1995), pp. 231-300.

[11] R. Simonton and A. F. Tasch, in Handbook of Ion Implantation Technology, edited by J.F. Ziegler (Elsevier, Amsterdam, 1992), pp. 119-221.

[12] M. Current, G. Hobler, Y. Kawasaki, and M. Sugitani, in Proceedings of the 22nd International Conference on Ion Implantation Technology (IEEE, New York, 2018), pp. 251-254.

[13] R. L. Davidchack and B. B. Laird, Phys. Rev. Lett. 94, 086102 (2005).

[14] A. Southern, W. R. Willis, and M. T. Robinson, J. Appl. Phys. 34, 153 (1963).

[15] H. E. Roosendaal, in Sputtering by Particle Bombardment I, edited by R. Behrisch (Springer, Berlin, 1981), pp. 219-256.

[16] D. E. Harrison, Jr., P. Kelly, B. J. Garrison, and N. Winograd, Surf. Sci. 76, 311 (1978).

[17] G. K. Wehner, J. Appl. Phys. 26, 1056 (1955).

[18] A. Litnovsky, Y. Krasikov, M. Rasinski, A. Kreter, C. Linsmeier, P. Mertens, B. Unterberg, U. Breuer, and T. Wegener, Fusion Eng. Des. 123, 674 (2017).

[19] H. Gnaser, Energy and angular distributions of sputtered species, in Sputtering by Particle Bombardment (Springer, New York, 2007), pp. 231-328.

[20] G. Betz and K. Wien, Int. J. Mass Spectrom. Ion Process. 140, 1 (1994).

[21] J. F. Ziegler, J. P. Biersack, and M. D. Ziegler, The stopping and range of ions in matter, SRIM, http://www.srim.org (2013).

[22] A. Mutzke, R. Schneider, W. Eckstein, and R. Dohmen, SDTrimsP: Version 5.00. ipp, report, (12/8), Max PlanckInstitut fr Plasmaphysik, 2011.

[23] C. Lehmann and P. Sigmund, Phys. Status Solidi B 16, 507 (1966).

[24] P. Sigmund, Phys. Rev. 184, 383 (1969).

[25] R. Arredondo, M. Oberkofler, T. Schwarz-Selinger, U. von Toussaint, V. Burwitz, A. Mutzke, E. Vassallo, and M. Pedroni, Nucl. Mater. Energy 18, 72 (2019).

[26] M. Hölscher, D. Raabe, and K. Lücke, Steel Res. 62, 567 (1991).
[27] A. Manhard, G. Matern, and M. Balden, Practical Metallogr. 50, 5 (2013).

[28] D. Onderdelinden, Can. J. Phys. 46, 739 (1968).

[29] G. Hobler, Nucl. Instrum. Methods Phys. Res., Sect. B 96, 155 (1995).

[30] See Supplemental Material at http://link.aps.org/ supplemental/10.1103/PhysRevLett.125.225502 for details and additional results of simulations and experiments, which includes Refs. [31-46].

[31] C. Ebm and G. Hobler, Nucl. Instrum. Methods Phys. Res., Sect. B 267, 2987 (2009).

[32] G. Hobler, D. Maciażek, and Z. Postawa, Phys. Rev. B 97, 155307 (2018).

[33] J. F. Ziegler, J. P. Biersack, and U. Littmark, The Stopping and Range of Ions in Solids (Pergamon Press, New York, 1985).

[34] D. Onderdelinden, Appl. Phys. Lett. 8, 189 (1966).

[35] O. S. Oen and M. T. Robinson, Nucl. Instrum. Methods 132, 647 (1976).

[36] M. P. Allen and D. J. Tildesley, Computer Simulation of Liquids (Oxford University Press, Oxford, England, 1989).

[37] M.-C. Marinica, L. Ventelon, M. R. Gilbert, L. Proville, S. L. Dudarev, J. Marian, G. Bencteux, and F. Willaime, J. Phys. Condens. Matter 25, 395502 (2013).

[38] A. E. Sand, J. Dequeker, C. S. Becquart, C. Domain, and K. Nordlund, J. Nucl. Mater. 470, 119 (2016).

[39] X. Yi, A. Sand, D. Mason, M. Kirk, S. Roberts, K. Nordlund, and S. Dudarev, Europhys. Lett. 110, 36001 (2015).

[40] A. E. Sand, J. Byggmästar, A. Zitting, and K. Nordlund, J. Nucl. Mater. 511, 64 (2018).

[41] J. Delley, J. Chem. Phys. 92, 508 (1990).

[42] DMol is a trademark of AccelRys. Inc.

[43] K. Nordlund, N. Runeberg, and D. Sundholm, Nucl. Instrum. Methods Phys. Res., Sect. B 132, 45 (1997).

[44] J. Jussila, F. Granberg, and K. Nordlund, Nucl. Mater. Energy 17, 113 (2018).

[45] D. Sprouster, R. Giulian, L. Araujo, P. Kluth, B. Johannessen, N. Kirby, K. Nordlund, and M. C. Ridgway, Phys. Rev. B 81, 155414 (2010).

[46] L. Zhong, J. Wang, H. Sheng, Z. Zhang, and S. X. Mao, Nature (London) 512, 177 (2014).

[47] M. T. Robinson, J. Appl. Phys. 54, 2650 (1983).

[48] M. Hautala and J. Likonen, Phys. Rev. B 41, 1759 (1990).

[49] A. V. Rogov, Y. V. Martynenko, N. E. Belova, and V. I. Shulga, Radiat. Eff. Defects Solids 166, 894 (2011).

[50] K. Nordlund, F. Djurabekova, and G. Hobler, Phys. Rev. B 94, 214109 (2016).

[51] K. Nordlund, Comput. Mater. Sci. 3, 448 (1995).

[52] H. M. Urbassek and J. Michl, Nucl. Instrum. Methods Phys. Res., Sect. B 22, 480 (1987).

[53] K. T. Waldeer and H. M. Urbassek, Nucl. Instrum. Methods Phys. Res., Sect. B 73, 14 (1993).

[54] K. Nordlund, K. O. E. Henriksson, and J. Keinonen, Appl. Phys. Lett. 79, 3624 (2001).

[55] S. Bouneau, A. Brunelle, S. Della-Negra, J. Depauw, D. Jacquet, Y. Le Beyec, M. Pautrat, M. Fallavier, J. Poizat, and H. Andersen, Phys. Rev. B 65, 144106 (2002). 
[56] G. Greaves, J. Hinks, P. Busby, N. Mellors, A. Ilinov, A. Kuronen, K. Nordlund, and S. Donnelly, Phys. Rev. Lett. 111, 065504 (2013).

[57] PARCAS Computer code, available open source at https:// gitlab.com/acclab/parcas/.

[58] K. Nordlund, M. Ghaly, R. S. Averback, M. Caturla, T. D. de La Rubia, and J. Tarus, Phys. Rev. B 57, 7556 (1998).

[59] M. Ghaly, K. Nordlund, and R. S. Averback, Philos. Mag. A 79, 795 (1999).

[60] J. Goulden, P. Trimby, and A. Bewick, Microsc. Microanal. 24, 1128 (2018).

[61] W. Pantleon, Scr. Mater. 58, 994 (2008).

[62] W. He, W. Ma, and W. Pantleon, Mater. Sci. Eng. A 494, 21 (2008).

[63] K. Schlueter and M. Balden, Int. J. Refract. Met. Hard Mater. 79, 102 (2019).

[64] K. Schlueter, M. Balden, and T. da Silva, Int. J. Refract. Met. Hard Mater. 88, 105189 (2020).

[65] H. S. Huang, C. H. Chiu, I. T. Hong, H. C. Tung, and F. S. S. Chien, Mater. Charact. 83, 68 (2013).
[66] T. Nagasaki, H. Hirai, M. Yoshino, and T. Yamada, Nucl. Instrum. Methods Phys. Res., Sect. B 418, 34 (2018).

[67] T. A. Runkler, Data Analytics: Models and Algorithms for Intelligent Data Analysis (Springer, New York, 2016).

[68] Y. Stark, R. Frömter, D. Stickler, and H. P. Oepen, J. Appl. Phys. 105, 013542 (2009).

[69] R. Franklin, E. Kirk, J. Cleaver, and H. Ahmed, J. Mater. Sci. Lett. 7, 39 (1988).

[70] L. Eriksson, J. A. Davies, and P. Jespersgaard, Phys. Rev. 161, 219 (1967).

[71] A. E. Sand, R. Ullah, and A. A. Correa, npj Comput. Mater. 5, 43 (2019).

[72] V. Yurasova and V. Eltekov, Vacuum 32, 399 (1982).

[73] J. Lindhard, Influence of Crystal Lattice on Motion of Energetic Charged Particles, Matematisk-fysiske Meddelelser Vol. 34 (The Royal Danish Society of Sciences, København (Copenhagen), 1965).

[74] G. Hobler, Radiat. Eff. Defects Solids 139, 21 (1996).

[75] P. Sigmund, Thin Solid Films 520, 6031 (2012).

[76] M. T. Robinson, in Sputtering by Particle Bombardment I, edited by R. Behrisch (Springer, Berlin, 1981), pp. 73-144. 